\title{
A Novel Technique for Simulating Transcranial Doppler Examinations In Vitro
}

\author{
Robin Hart', Philip D. Hart ${ }^{2}$ and Stuart Bunt ${ }^{1}$ \\ ${ }^{1}$ Department of Anatomy and Human Biology, University of Western Australia \\ ${ }^{2}$ The Hart Combination Company Limited, Bath, United Kingdom
}

\begin{abstract}
The left internal carotid artery of a cadaveric head was injected with a methyl methacrylate casting resin. The cast of the vessels of the Circle of Willis was used to construct an artificial arterial system from Tygon R3603 tubing. Sylgard 527 elastomer gel was used to replace the intracranial contents. Sylgard 184 was used to replicate the extracranial tissues. The model was perfused with a fluid consisting of sephadex particles and glycerine, which approximates normal blood characteristics. Physiologically accurate fluid flow was achieved using a custom designed computer controlled syringe pump which could deliver normal or pathological flow profiles as required. Age and sex matched control data were compared with data obtained from the model system using a Transcranial Doppler scanner. The results showed that a close approximation between the artificial and in vivo flow patterns could be achieved by manipulation of the flow profile.
\end{abstract}

\section{Introduction}

Transcranial Doppler Ultrasound (TCD) is a diagnostic technique used to assess blood flow in the head. This is important in the management of cerebral vasospasm (VSP), a condition commonly seen in survivors of acute subarachnoid haemorrhage. The detection of VSP is most accurately performed using Digital Subtraction Angiographic studies, but such invasive procedures carry high inherent risk, and may be contraindicated. TCD, although less sensitive and specific, poses significantly less risk to the patient [1].

Transcranial Doppler ultrasound suffers two significant limitations:

a. It is a conceptually difficult technique to learn and demonstrate; and

b. Intra-operator variability may lead to high rates of false positive or false negative results [1].

This study aims to construct an artificial system which is able to simulate TCD examinations of both normal and pathologic vascular states. Training in TCD examination technique can then be performed in a controlled environment. This study forms the first part of the development of a system of co-registration between digital subtraction angiography images of vascular anatomy, and physiological data from TCD examinations. 


\section{Methods}

\subsection{Preparation of the Cadaveric Model}

A 74 year old female cadaver was cannulated through the left internal carotid artery, and the cerebral circulation flushed using normal saline. An injection of $50 \mathrm{mls}$ of green methyl methacrylate casting material ("Vertex" Dentimex Zeist, Holland) was then made using a 12 french arterial catheter, with hand injection from a $50 \mathrm{ml}$ syringe [2]. The arterial catheter was withdrawn, the wound closed, and the body embalmed for three months via cannulation of the femoral artery and infusion of embalming solution (Formalin (7.5\%), Glycerin (20\%), Phenol (6\%), Methylated Spirit (40\%), Water (26.5\%)).

Specimen Dissection. After removal of the head at the fourth cervical vertebra, a plaster cast impression of the head was taken. The postero-superior part of the skull was then removed at its widest diameter. The extracranial tissues were grossly dissected, and the cervical spine removed.

All brain tissues, including the arachnoid mater, were removed. The Circle of Willis (COW) was left in situ, and sectioned to remove all arteries except: the distal internal carotid; anterior, middle and posterior cerebral (ACA, MCA and PCA respectively); anterior ( $\mathrm{ACoA}$ ) and posterior (PCoA) communicating, vertebral (VA), basilar (BA) and cerebellar arteries. The vessel walls were completely dissected to reveal the previously injected Vertex casting medium, which was removed from the skull, and immersed in saturated potassium hydroxide for three days.

Vessel Reconstruction. The vertex cast of the vessels was used as a model from which an artificial circulation was constructed, using direct measurement, from Tygon R3603 tubing (Lab Supplies Australia). This material has some elastic properties, although there was no attempt to closely replicate in vivo arterial elasticity. This model was then re-inserted into the skull.

A de-aerated silicone based gel, Sylgard 527 dielectric polymer (Dow Corning), was used to replace the intracranial contents. All air was removed by placement under vacuum of 29 inches of mercury for 30 minutes This material is recognised as possessing physical and ultrasonic properties similar to those of brain tissue [3,4].

After a seven day delay for cure, a second polymer gel, Sylgard 184 (Dow Corning) was mixed and de-aerated. This was then poured around the skull assembly, previously placed in the facial cast, to form a robust seal, which also recreated the facial features of the cadaver. 


\subsection{Perfusion}

Pump Construction. The pump consists of a $12 \mathrm{ml}$ capacity, $12 \mathrm{~mm}$ stroke piston and cylinder assembly, driven by a stepper motor. The cylinder head has an inlet and an outlet ball valve, both being closed by a compression spring. The outlet valve closing spring has a tension adjustment screw to vary the opening and closing pressures. The output of the pump was designed to allow emulation of a typical maximum cerebral supply of 1 litre of perfusate per minute.

The stepper motor was driven by a drive card (GSM5, Greenwich Instruments), controlled by a $300 \mathrm{MHz}$ Pentium ${ }^{\circledR}$ personal computer. With a step angle of 1.8 degrees, the pump has 330 steps in the full travel of the piston.

Software. A program was written in Visual Basic $6^{\odot}$ (Microsoft) which allowed digitised data from in vivo flow conditions to be delivered to the pump. Up to seven different waveforms may be previously defined, and these may be combined in any order to a maximum of thirteen in any one wave train. This train is self-repeating. Data plots of normal left ventricular volume against time were obtained [5]. These were manually digitised, using graph paper, with sample points at $25 \mathrm{~ms}$ intervals. Data points were plotted as percentage of maximal contraction against time. These were converted to the appropriate number of motor steps. This output was delivered to the program at pulse rates of 30 to 180 beats per minute (bpm).

Delivery System. The delivery system was a development of previous work $[6,7,8,9]$. The pump output was taken from the outlet valve via vinyl laboratory tubing (Nylex, Australia) of $8 \mathrm{~mm}$ internal diameter. This tube fed into the middle of a capacitance tank holding $1 \mathrm{~L}$ of perfusate. The outlet from the tank was in the middle of the vessel on the side opposite the inlet. A baffle plate was set vertically from the bottom of the tank dividing the line between the inlet and the outlet to ensure that the smoothing effect of the tank was not eliminated by direct streaming between inlet and outlet. At maximal filling, the additional pressure head provided by the tank was $5 \mathrm{~cm}$.

The $8 \mathrm{~mm}$ outlet from the tank terminated in a four-way flow divider. The output from the divider was taken via $5 \mathrm{~mm}$ tubing to the inlet ports of the VAs and ICAs of the model. The differential flow through each of these tubes was controlled by a tap. The system was set to deliver $82 \%$ through the anterior circulation, and $18 \%$ through the posterior circulation. This simulates the typical in vivo distributions [10].

\subsection{Perfusate}

A perfusate with the same effective acoustic reflector surface as blood was developed to provide return echo signals from which doppler data was derived. The perfusion medium was prepared using the following concentrations of materials:

Glycerol:

Sephadex particles ( $80 \mu \mathrm{m}$ average particle size)

Distilled water 
The glycerol allows sufficient reduction in surface tension of the water to allow perfusion of the models. The concentration of sephadex particles allowed the use of identical gain settings on the TCD scanner for both in vitro and in vivo examinations.

\subsection{Ultrasound Scanning}

Model. The model was subjected to a TCD examination of all major arterial segments accessible from the trans-pterional window: ICA, M1 and M2 MCA segments, A1 ACA and P1 PCA segments, using an EME Companion (Nicolet Biomedical, Madison, WI, USA) single channel $2 \mathrm{MHz}$ TCD scanner. Trans-orbital access was used for the ophthalmic artery and carotid artery siphon. These examinations were undertaken while the model was perfused with a normal cardiac output wave. The TCD data (Table 1) were taken from the insonation attempt which yielded the best signal:noise ratio for each vessel segment. Controls were sidematched scans of age and sex matched patients, with normal cerebral circulation. Figures 1 and 3 were obtained from the MCA with the sample volume at a depth of $60 \mathrm{~mm}$ for both in vivo and in vitro cases.

Gel Samples. The gels used do not have the identical behaviour when insonated as the tissues that they replace. A sample of each was prepared to generate correction factors. These correction factors take into account the difference in transmission speed of ultrasound through the gel and tissue, and hence the difference between real and apparent depth measurements.

A degassed sample of 527 gel was poured into a cylindrical container measuring $75.5 \mathrm{~mm}$ deep, and $18 \mathrm{~mm}$ in diameter. Degassed 184 gel was poured into a cuboid container, $44.5 \mathrm{~mm}$ deep, $34 \mathrm{~mm}$ wide and $53 \mathrm{~mm}$ long. All measurements taken with vernier calipers. To measure the apparent depth of the gel samples (the TCD system being unable to produce an image) the sample of both 527 and 184 gel were imaged with a Toshiba PowerVision ultrasound system. A small quantity of Scan ultrasound coupling medium (Parker Laboratories Inc., Fairfield, N.J., USA) was used between a 7.5 MHz linear array ultrasound probe and the surface of the gel sample. As speed of transmission of ultrasound through a given medium is frequency independent, these results are also comparable to those of a $2 \mathrm{MHz}$ TCD probe. The samples were insonated from the exposed gel surface at the top of the container, and measurements taken between this gel surface and the bottom of the container, using the on-screen cursors supplied as part of the Toshiba ultrasound system software (Table 2). These are accurate to $\pm 0.1 \mathrm{~mm}$ (Toshiba). 


\section{Results}

\subsection{Model}

The model demonstrated circulatory tubal patency, and successful perfusion at rates of up to $900 \mathrm{mls} / \mathrm{min}$ was achieved.

\subsection{Waveform Data}

Figures $1 \mathrm{a}$ and $1 \mathrm{~b}$ show that the artificial circulation closely mimics actual flow. The waveform shown in Figure 1a was generated using the pump input shown in Figure $2 \mathrm{a}$, applied at a rate of 30 beats per minute. There is, however, considerable angularity of the wave shape, with elements resembling square wave functions seen throughout the profile. In contrast, the in vivo waveform demonstrates a smooth profile.

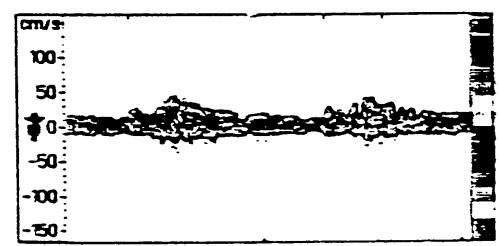

Fig. 1a. Artificial TCD Output

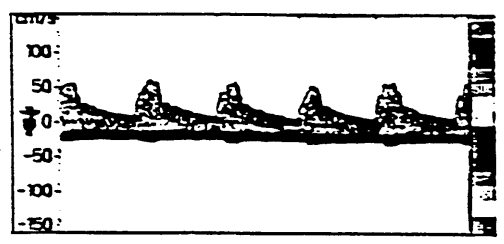

Fig. 1b. In Vivo TCD Output

Figure 1a shows the TCD output with a sample depth of $60 \mathrm{~mm}$. This equates to a real depth of $59.76 \mathrm{~mm}$ (See Table 2). This is within the sample volume size of $15 \mathrm{~mm}$, and hence the error can be ignored. Figure $1 \mathrm{~b}$ illustrates a normal control graph from a 73 year old female. This waveform represents a heamodynamic state in the same anatomic location

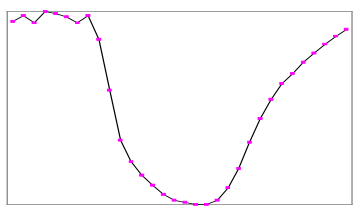

Fig. 2a Normal pump output

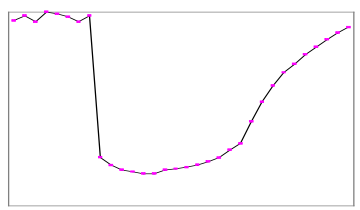

Fig. 2b Modified pump output

Figure 2 shows input waveforms to the pump, as piston position (\% of travel) against time. Figure 2a illustrates a normal left ventricular contraction profile. Figure $2 \mathrm{~b}$ shows a modified input waveform with the systolic contraction more rapid, and the time at contraction lengthened

\footnotetext{
${ }^{1}$ These are exact replicas of the original TCD scanner output
} 
A modification of the wave supplied to the pump (Figure 2b), and an increase in the pump rate to 62 beats per minute, led to the results shown by Figures $3 \mathrm{a}$ and $3 \mathrm{~b}$.

The altered (non-physiologic) input waveform, when combined with the artificial circulation, yields a hybrid circulation which has the effect of smoothing the TCD output, and hence closely approximating a true in vivo TCD output wave profile.

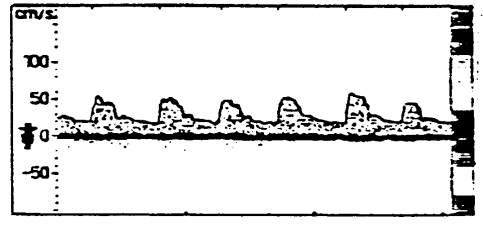

Fig. 3a. Modified artificial TCD Output

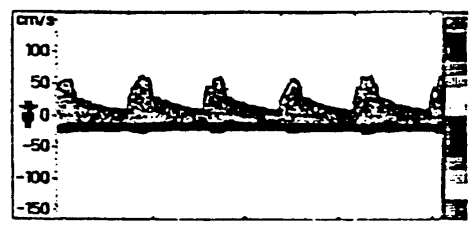

Fig. 3b In Vivo TCD output

Figure $3 \mathrm{a}$ illustrates the TCD output using the modified pump waveform, and Figure $3 \mathrm{~b}$ illustrates the age-matched control at the same depth ${ }^{1}$

Table 1. Data comparing the waveforms shown in Figure 1 and Figure 3

\begin{tabular}{|c|c|c|c|c|c|c|}
\hline & & $\begin{array}{l}\text { Mean } \\
\text { Velocity }\end{array}$ & $\begin{array}{l}\text { Systolic } \\
\text { Velocity }\end{array}$ & $\begin{array}{l}\text { Diastolic } \\
\text { Velocity }\end{array}$ & P.I & R.I \\
\hline \multirow[t]{2}{*}{ Figure 1} & $\begin{array}{l}\text { In Vitro } \\
\text { (Fig. 1a) }\end{array}$ & $21 \mathrm{~cm} / \mathrm{s}$ & $43 \mathrm{~cm} / \mathrm{s}$ & $10 \mathrm{~cm} / \mathrm{s}$ & 1.43 & 0.77 \\
\hline & $\begin{array}{l}\text { In Vivo } \\
\text { (Fig. 1b) }\end{array}$ & $34 \mathrm{~cm} / \mathrm{s}$ & $70 \mathrm{~cm} / \mathrm{s}$ & $18 \mathrm{~cm} / \mathrm{s}$ & 1.49 & 0.75 \\
\hline \multirow[t]{2}{*}{ Figure 3} & $\begin{array}{l}\text { In Vitro } \\
\text { (Fig. } 3 a)\end{array}$ & $22 \mathrm{~cm} / \mathrm{s}$ & $48 \mathrm{~cm} / \mathrm{s}$ & $9 \mathrm{~cm} / \mathrm{s}$ & 1.63 & 0.81 \\
\hline & $\begin{array}{l}\text { In Vivo } \\
\text { Fig. } 3 b)\end{array}$ & $34 \mathrm{~cm} / \mathrm{s}$ & $70 \mathrm{~cm} / \mathrm{s}$ & $18 \mathrm{~cm} / \mathrm{s}$ & 1.49 & 0.75 \\
\hline
\end{tabular}

P.I. = Pulsatility Index, R.I. = Resitivity Index

In all cases the blood flow velocity of the in vitro circulation is between one half and two thirds of that in vivo. This may be due inertial effects of the pump mechanism, and differences in the compliance of the vessels between the pump and model

Table 2. Data showing the derivation of ultrasound depth correction factors for the gels

\begin{tabular}{|l|l|l|}
\hline & Sylgard 527 Gel & Sylgard 184 Gel \\
\hline Sample depth (mm) & 75.5 & 44.5 \\
\hline Measured depths (mm) & $65.4,66.1,66.9,67.3$ & $67.9,68.0,67.6,67.6$ \\
\hline = Average (mm) & 66.4 & 67.8 \\
\hline Measured /real depth (\%) & 88 & 152 \\
\hline
\end{tabular}

From these data, a table (Table 3) was generated to allow correction of scanning depth errors. 
Table 3. Correction table for different skin thicknesses (Sylgard 184) over typical insonation depths

\begin{tabular}{|l|l|l|l|l|l|l|}
\hline \multirow{2}{*}{$\begin{array}{c}\text { Real Depth } \\
\text { nm) }\end{array}$} & \multicolumn{6}{|l|}{ Skin Thickness $(\mathbf{m m})$} \\
\cline { 2 - 7 } & 4.00 & 6.00 & 8.00 & 10.00 & 12.00 & 14.00 \\
\cline { 2 - 7 } & \multicolumn{5}{|l|}{ Error $(\mathbf{m m})$} \\
\hline 40 & -1.66 & -0.40 & 0.86 & 2.12 & 3.38 & 4.64 \\
\hline 44 & -1.88 & -0.62 & 0.64 & 1.90 & 3.16 & 4.42 \\
\hline 48 & -2.10 & -0.84 & 0.42 & 1.68 & 2.94 & 4.20 \\
\hline 52 & -2.32 & -1.06 & 0.20 & 1.46 & 2.72 & 3.98 \\
\hline 56 & -2.54 & -1.28 & -0.02 & 1.24 & 2.50 & 3.76 \\
\hline 60 & -2.76 & -1.50 & -0.24 & 1.02 & 2.28 & 3.54 \\
\hline 66 & -4.74 & -3.48 & -2.22 & -0.96 & 0.30 & 1.56 \\
\hline 70 & -5.18 & -3.92 & -2.66 & -1.40 & -0.14 & 1.12 \\
\hline 76 & -5.84 & -4.58 & -3.32 & -2.06 & -0.80 & 0.46 \\
\hline 80 & -6.28 & -5.02 & -3.76 & -2.50 & -1.24 & 0.02 \\
\hline
\end{tabular}

\section{Discussion}

Alteration of both the wave profile and the rate of the pump input has a smoothing effect on the TCD output. The lack of a real circulation (particularly small vessels), the lack of elastic capacitance vessels, and the relatively high inertia of the pump, combine to produce a circulatory system very different from that in the human subject. The use of an artificial pump input is required to emulate a normal output wave. Further refinement of the vessels of the circulation may lead to a closer approximation to the real situation.

There is close correlation between the resistivity $(0.77$ versus $0.75=2.5 \%)$ and pulsatility $(1.43$ versus $1.49=4 \%)$ indices of the real and artificial waveforms in the input wave shown in Figure 2a. For the modified input waveform shown in Figure $2 \mathrm{~b}$, these values are slightly worse: resistivity: 0.81 versus $0.75=9 \%$; pulsatility: 1.63 versus $1.49=9 \%$. However, this input wave yields a closer output wave shape. This may reflect the artificial nature of the circulation, particularly the lack of peripheral resistance vessels.

This study confirms that Sylgard 527 is a useful tissue analogue for brain. In combination with Sylgard 184, Table 3 demonstrates that sample depth errors lie within the resolution limit of current TCD scanners (typically $2 \mathrm{~mm}$ ), when imaging through typical tissue thicknesses. 


\section{Conclusion}

This study describes a technique to create anatomically accurate models of the cerebral circulation from cadaveric material. The apparatus described allows these models to be perfused in a way that can simulate in vivo flow within the resolution limits of the TCD technique. Future studies will reveal whether the restrictions imposed by the artificial nature of the system can be overcome to allow accurate replication of other in vivo flow profiles.

\section{Acknowledgments}

This work was in part supported by a grant from the Medical Research Foundation of Royal Perth Hospital; and by the Lotteries Commission of Western Australia. The authors wish to thank Dr Mark Kanghure, Ms Helen Wines, and Mrs Elvie Haluszkiewicz (Royal Perth Hospital Radiology Department); Mr Darryl Kirk, Mr Mark Peterson Mr Brian Peppler and Mr Albert Kalajzich (Department of Anatomy and Human Biology, University of Western Australia).

\section{References}

1. Babikian, V.L., Weschler, L.R.: Transcranial Doppler Ultrasonography. Mosby Year Book, St Louis (1993)

2. Kerber, C.W., Heilman, C.B.: Flow Dynamics in the Human Carotid Artery: 1. Preliminary Observations using a Transparent Elastic Model. A.J.N.R. 13 (1992) 173-180

3. Meaney, D.F., Smith, D.H., Shreiber, D.I., Allison, C.B., Miller, R.T., Ross, D.T., Gennarelli, T.A.: Biomechanical Analysis of Experimental Diffuse Axonal Injury J. Neurotrauma 124 (1995) 689-694

4. Kerber, C.W., Heilman, C.B., Zanetti, P.H.: Transparent Elastic Models 1: A Brief Technical Note. Biorheology 26 (1989) 1041-1049

5. Anthony, C.P., Thibodeau, G.A., Preszbindowski K.S.: Textbook of Anatomy and Physiology. The C.V. Mosby Company, St Louis (1979)

6. Lutz, R.J., Hsu, L., Menawat, A., Zrubek, J., Edwards, K.: Comparison of Steady and Pulsatile Flow in a Double Branching Arterial Model. J. Biomechanics 16 (1983) 753-766

7. Roach, M.R., Scott, S., Ferguson, G.G.: The Hemodynamic Importance of the Geometry of Bifurcations in the Circle of Willis (Glass Model Studies). Stroke 3 (1972) 255-267

8. Stehbens, W.E.: Turbulence of Blood Flow. Quart. J. Exp. Physiol. 44 (1959) 110-117

9. Cohen, M.I., Wang, D.-M., Tarbell, J.M.: Measurement of Oscillatory Flow Pressure Gradient in an Elastic Artery Model. Biorheology 324 (1995) 459471

10. Boyajian, R.A., Schwend, R.B., Wolfe, M.M., Bickerton, R.E., Otis, S.M.: Measurement of Anterior and Posterior Circulation Flow Contributions to Cerebral Blood Flow: An Ultrasound-Derived Volumetric Flow Analysis. J. Neuroimag. 5 (1995) 1-3 